International Journal of Pure and Applied Mathematics

Volume 82 No. 5 2013, 709-718

ISSN: 1311-8080 (printed version); ISSN: 1314-3395 (on-line version)

url: http://www.ijpam.eu

doi: http://dx.doi.org/10.12732/ijpam.v82i5.4

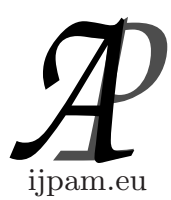

\title{
SUDOKU: THE NEW SMASH HIT PUZZLE GAME
}

\author{
Yutaka Nishiyama \\ Department of Business Information \\ Faculty of Information Management \\ Osaka University of Economics \\ 2, Osumi Higashiyodogawa Osaka, 533-8533, JAPAN
}

\begin{abstract}
Sudoku is now popular in many countries. This article explains the history and mathematics behind Sudoku. This includes the basic rules of Sudoku, a trial-and-error solution method, a discussion of the number of patterns completed, minimum Sudoku, and the application of Euler's Latin squares. The popularity of Sudoku in the UK is also discussed.
\end{abstract}

AMS Subject Classification: 11A02, 00A09, 97A20

Key Words: Sudoku, number-place, minimum Sudoku, Latin squares

\section{A Sudoku Book on the Best Seller List}

In 2005 I had the chance to spend a year abroad conducting research at Cambridge University, and around May last year, one of my colleagues from England sent me the following enquiry: "Is there a general solution method for the type of Japanese puzzle known as Sudoku?" I tried to remember if I had heard of such a puzzle, and after thinking about it for a while I remembered that in the

Received: October 13, 2012

(c) 2013 Academic Publications, Ltd. url: www.acadpubl.eu 
UK they pronounced it 'su doh ku' rather than 'suudoku'. Thinking about it some more, I remembered seeing some of the tired office workers on the train in Japan pitting themselves against this puzzle as if possessed. Some people regarded it as a slightly unhealthy puzzle hobby, and I had not once tried it. At that point I didn't even know the correct name for it.

Sudoku, written

\section{数独}

in Japanese, is the name used in the magazine pazuru tsushin nikori, and is pronounced 'suu do ku'. Sudoku is a contraction of a phrase meaning 'numerals remaining alone', and has been popular in Japan since around 1986. The puzzle had a previous incarnation in a puzzle magazine published in New York in the 1970s by the company Dell (no relation to the computer company of the present day). It was popular under the name 'number-place', which refers to the placing of numerals, and subsequently became popular in Japan under the alternative name, Sudoku. It is currently gaining in popularity in many countries.

I was rather delighted that it was not being called 'number-place', but instead the Japanese name, Sudoku. I investigated the extent to which people are familiar with this puzzle in the UK. As the result of British broadsheet newspapers such as 'The Times' and 'The Guardian' taking up Sudoku around spring last year, all at once more general newspapers and community pages started publishing Sudoku problems as well. There are now books of problems that are published by these newspaper companies (see Figure 1.) In Japanese terms, this corresponds to the Yomiuri shinbun or Asahi shinbun newspapers releasing books of Sudoku puzzles. If you went to a book store you would find a Sudoku book on the top of the best-sellers shelf. Once when I was watching a program on a certain TV station there was an interview with a celebrity, who was asked "Do you enjoy Sudoku?" and replied "Of course!"
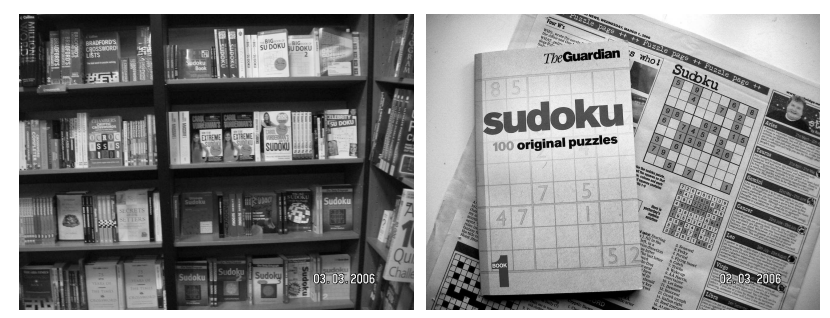

Figure 1: The sudoku corner of the shelves in a bookshop (left). Standalone book and column in the Guardian (right) 
The woman who lived next door to me enjoyed solving the Sudoku problem in her daily newspaper, and told me that it had become part of her daily routine. It seems as though people solving Sudoku had a certain glow.

One of the topics at a mathematics exhibition in July at a huge shopping center in Newcastle in the UK was a Sudoku corner. It was organized by the staff from a mathematical education research laboratory, who had prepared a complete set of Sudoku teaching materials. I was surprised that they had gone as far as producing educational resources. On the train back to Cambridge there were students earnestly wrestling with puzzle books. When I looked to see what puzzle they were working on, it was Sudoku. Recently there have also been Sudoku for game consoles, and I have seen people on buses enjoying Sudoku in such a way. I will touch upon it again below but Sudoku, unlike crossword puzzles, may be suited not to pencil and paper, but rather to personal computers.

Making it this far, Sudoku has really boomed. I made a pun in Japanese by joking that the 'doku' part means 'poison' since people who are addicted to it find they cannot escape, but I didn't manage to raise a laugh in the UK.

\section{The Mathematics of Sudoku}

There are many unexpected and hidden fans of Sudoku, but for the benefit of first-timers I'll explain the rules and a simple solution method.

The basic rules of Sudoku. Sudoku is one of those puzzles that involves filling in the numerals from 1 to 9 , in a $9 \times 9$ square grid partitioned into $3 \times 3$ blocks. The thing is to place the numerals in such a way that no $3 \times 3$ block, nor any row nor column contains a duplicated numeral. The derivation of the name Sudoku, from 'numerals remaining alone' is well chosen, and refers to the fact that since duplications are forbidden, the numerals must remain alone. Of the $9 \times 9=81$ squares in the grid shown in Figure 2, 30 of them already have a number shown. These numbers are taken as hints and the remaining spaces are filled in. These hints may be given in a symmetrical or an asymmetrical fashion. The hints in Figure 2 are symmetrical. (see the Sudoku article on Wikipedia.) [4]

Trial-and-error solution method. In the example, starting from the top, the first row already has a 5 in it so another 5 cannot be written in this row. The second row also already has a 5 in it, and the first column on the right-hand side already has a 5 in it so that neither this row nor column can take another 5 . Elimination lines meaning that a 5 cannot be placed in these 


\begin{tabular}{|l|l|l|l|l|l|l|l|l|}
\hline 5 & 3 & & & 7 & & & & \\
\hline 6 & & & 1 & 9 & 5 & & & \\
\hline & 9 & 8 & & & & & 6 & \\
\hline 8 & & & & 6 & & & & 3 \\
\hline 4 & & & 8 & & 3 & & & 1 \\
\hline 7 & & & & 2 & & & & 6 \\
\hline & 6 & & & & & 2 & 8 & \\
\hline & & & 4 & 1 & 9 & & & 5 \\
\hline & & & & 8 & & & 7 & 9 \\
\hline
\end{tabular}

Figure 2: An example sudoku

two rows or this column can therefore be drawn. Then when looking at the grid in terms of $3 \times 3$ blocks, the upper right block already contains a 6 so there is only one free box which can, and therefore must contain a 5 . The box in the 3rd row and 7th column is thus filled in (Figure 3).

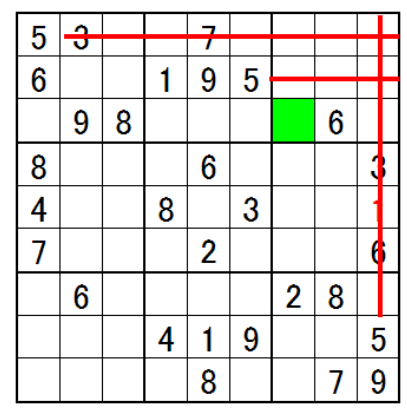

Figure 3: Solution example (the position of a 5 is determined)

By means of this process the numbers are filled in according to trial-anderror, but it's difficult to prove mathematically what kind of method is optimal. From experience, making use of the numbers filled in as hints and focusing on the places which already have many boxes filled in makes for a quick solution. When 8 of the 9 locations of a numeral are filled in, there is only one possible location left so its entry is fixed. When 7 locations are filled in it is easier to find the answer than when 6 locations are filled in. From the hints in Figure 3 , there are 5 locations containing an 8 , and 2 locations containing a 2 , so it is more efficient to start from the 8 . 
The number of patterns that can be generated. When all the numerals are filled in, the result looks like Figure 4. This is the completed pattern of the Sudoku. For each of the 9 rows, each of the 9 columns and each of the $3 \times 3$ blocks, there is no duplication of the numerals from 1 to 9 .

\begin{tabular}{|l|l|l|l|l|l|l|l|l|}
\hline 5 & 3 & 4 & 6 & 7 & 8 & 9 & 1 & 2 \\
\hline 6 & 7 & 2 & 1 & 9 & 5 & 3 & 4 & 8 \\
\hline 1 & 9 & 8 & 3 & 4 & 2 & 5 & 6 & 7 \\
\hline 8 & 5 & 9 & 7 & 6 & 1 & 4 & 2 & 3 \\
\hline 4 & 2 & 6 & 8 & 5 & 3 & 7 & 9 & 1 \\
\hline 7 & 1 & 3 & 9 & 2 & 4 & 8 & 5 & 6 \\
\hline 9 & 6 & 1 & 5 & 3 & 7 & 2 & 8 & 4 \\
\hline 2 & 8 & 7 & 4 & 1 & 9 & 6 & 3 & 5 \\
\hline 3 & 4 & 5 & 2 & 8 & 6 & 1 & 7 & 9 \\
\hline
\end{tabular}

Figure 4: The completed pattern

Look at the completed figure, in which the numerals are distributed evenly without any duplications. Don't you perceive a certain beauty here? How big is the number of such duplication-free distributions of the numerals? I supposed that it would probably be small, but investigating, I realized that a considerable number of patterns are possible. For example, for a small square grid of no more than $4 \times 4$ partitioned into $2 \times 2$ blocks, calculating the number of possible patterns revealed that there are 288. It is predictable that for $9 \times 9$ Sudoku problems, the number of patterns grows larger still. People have in fact calculated the number. Felgenhauer and Jarvis it as

$$
6,670,903,752,021,072,936,960 \approx 6.671 \times 10^{21}
$$

according to the formula

$$
9 ! \times 72^{2} \times 2^{7} \times 27,704,267,971
$$

(see Felgenhauer and Jarvis, 2005).[2] The last term in the formula is a prime number. The fact that it is a prime means that it cannot be factorized, so this is the most refined form in which the numerical formula can be expressed. I have not verified for myself whether or not the value of this formula is correct, but it surely is. It means that a considerable number of patterns can be formed, and it seems unlikely that all the Sudoku problems will be exhausted. 
Solution uniqueness. As I mentioned, Sudoku is a puzzle which involves writing numbers in the $9 \times 9=81$ squares of a grid. Some numbers are already shown as hints, and the number of hints is usually in the range of 20 to 36 . In the case of Figure 4, there are 30 hints, which leads to the unique solution shown in Figure 4. If the number of hints is small, then the smaller it is the more likely alternative solutions become. As extreme examples, if all of the $9 \times 9=81$ boxes are empty, then there is an almost unlimited number of solutions, but if 80 boxes are filled in then there is no scope for an alternative solution.

But what is the smallest number of hints that must be given in order to prevent alternative solutions? Although it does not constitute a proper mathematical proof, among those presented so far the smallest has been an asymmetrical Sudoku with 17 hints. Among the symmetrical Sudoku, the smallest has 18 hints.

There is an example of such a smallest 17-hint asymmetrical problem shown in Figure 5. This was made public on the internet, and is from a set of 450 smallest Sudoku problems revealed by Royle (see Royle, 2005). [3] There are few hints so it is difficult to solve, but the unique solution is fixed.

\begin{tabular}{|l|l|l|l|l|l|l|l|l|}
\hline & & & & & & & 3 & 1 \\
\hline 6 & & & & 2 & & & & \\
\hline & & & & 7 & & & & \\
\hline & 5 & & 1 & & 8 & & & \\
\hline 2 & & & & & & 6 & & \\
\hline & & & 3 & & & & 7 & \\
\hline & & & & 4 & & 2 & & \\
\hline & 3 & & 5 & & & & & \\
\hline 7 & & & & & & & & \\
\hline
\end{tabular}

Figure 5: Example smallest sudoku (asymmetrical, $\mathrm{n}=17$ )

Application of Euler's Latin squares. The origin of Sudoku and numberplace reach as far back as the Latin squares devised by the 18th century Swiss mathematician, Euler.

A Latin square has no duplicated numbers in its rows nor its columns. In Figure 7, the numbers from 1 to 4 are filled-in in each row, and each column also has the numbers from 1 to 4 , without duplications. A $4 \times 4$ Latin square is partitioned into $2 \times 2$ blocks. Adding the further condition that all the numbers from 1 to 4 must also be written in every block without duplications defines a Sudoku. Sudoku can therefore be regarded as an application of Latin squares. 
Latin Square

\begin{tabular}{|llll|}
\hline 1 & 2 & 3 & 4 \\
\hline 2 & 1 & 4 & 3 \\
\hline 3 & 4 & 1 & 2 \\
\hline 4 & 3 & 2 & 1 \\
\hline
\end{tabular}

\begin{tabular}{|ll|ll|}
\hline 1 & 2 & 3 & 4 \\
3 & 4 & 1 & 2 \\
\hline 4 & 3 & 2 & 1 \\
2 & 1 & 4 & 3 \\
\hline \multicolumn{4}{|c}{ Sudoku }
\end{tabular}

Figure 6: Latin squares and Sudoku

In general, an $\mathrm{n}^{2} \times \mathrm{n}^{2}$ Latin square can be divided into $\mathrm{n} \times \mathrm{n}$ blocks, and made into a $S u d o k u$ problem. The $9 \times 9$ grid is popular for $S u d o k u$, but at a higher level there are also $16 \times 16$ sized problems.

So Sudoku and Latin squares both involve many mathematical elements. They are not restricted to puzzles, and the following problems, which are convenient for university entrance examinations, can be constructed. With a $4 \times 4$ grid, how many Latin squares, and how many Sudoku grids are possible? The answer is that there are $4 ! \times 3 ! \times 4=576$ Latin squares, and $4 ! \times 2 ! \times 6=288$ Sudoku grids. This can be solved without a computer, using pencil and paper. Interested readers, please calculate the results!

Now, Euler demonstrated that the number of Latin squares with $\mathrm{n}=5$ is $5 ! \times 4 ! \times 56(1782)$. In relation to Sudoku grids, Bammel and Rothstein demonstrated that for Latin squares with $\mathrm{n}=9$, the number of Latin squares is

$$
9 ! \times 8 ! \times 377,597,570,964,258,816
$$

(see Bammel and Rothstein, 1975).[1] For the calculation, a computer of those times was used, namely, a PDP-10. Calculating the actual value of this formula yields

$$
\begin{gathered}
5,524,751,496,156,892,842,531,225,600 \\
\approx 5.525 \times 10^{27}
\end{gathered}
$$

When $\mathbf{n}=9$, the number of Latin squares is of the order $10^{27}$, and the number of Sudoku grids is of the order $10^{21}$, as shown above. About $10^{-6}$ of Latin squares are Sudoku grids, and they can be understood as further constrained patterns. Even so, the fact remains that there is an astronomical number of as many as $10^{21}$.

For both Latin squares and Sudoku grids, the arrangement of numerals is truly balanced. Focusing on this well-balanced aspect, Latin squares already 
have an application in the field of experimental design methodology. Perhaps Sudoku arrangements will also find an application in statistics and open up a new field of research. They are promising for the future. By this token, I also attempted the challenge of Sudoku in England. I reached a level at which, no matter what the problem, I could solve it given enough time. Sudoku is certainly a type of puzzle that supplies one with a sense of achievement. Perhaps this is due to the quality of the numerals' balance. I also suspect that unlike crossword puzzles, they are not oriented towards the pencil and eraser style. This is because difficult problems involve many hypotheses regarding the arrangement of numerals, and upon reaching a dead-end it is not clear how far to back-track. Maybe these puzzles are suited to computers after all.

\section{The Healthy Playful Psychology of the British}

I thought that in Japan Sudoku was only popular among puzzle fans, but in the UK, the whole nation seems to enjoy Sudoku. I wonder why this is? I thought about where this difference might lie. By way of a similar kind of puzzle to Sudoku, the UK is the birthplace of crosswords. Amateur detective novels such as those of Agatha Christie and Conan Doyle were also born in the UK. It occurred to me that perhaps having plenty of rain and overcast weather, it might be a climate-related national characteristic that people discovered the pleasure of puzzles and mystery novels while spending much time indoors.

Besides the many people who are fond of Sudoku in Europe, including the UK, there are also many mathematicians researching Sudoku. Even as a research theme Sudoku are not looked upon coldly, and many commendable pieces of research have appeared. In Japan, if one became absorbed in research on magic square or other moth-eaten problems, these being among the interests of math fanatics, such researchers would probably be labeled as 'not mathematicians'.

The population of the UK is about 60 million, which is half that of Japan. From a biological perspective it would not be unreasonable for there to be twice as many excellent researchers in Japan. From the industrial revolution in the 18th century to the peak of the Victorian era, wasn't the UK's greatest achievement these mechanism themselves? The historical process behind the success of the British Empire, by which much wealth was obtained from colonies in Asia and Africa, must not be forgotten, but there is an awareness that the country was developed though science and technology with mathematics as a prime example, and even now mathematics and mathematicians are held in high 
regard. Also, possessing the versatile language of English was an advantage. Japanese lacks versatility because it is difficult to achieve abundant literacy.

This generation in the UK turned out many scientists such as Newton (differential calculus), Napier (logarithms), Boole (Boolean algebra), Cavendish (physics), Maxwell (electromagnetism), Faraday (chemistry), and Darwin (evolution). Even today, researchers gather from all over the world at the Cavendish Research Laboratory in Cambridge. Cambridge has educated 70 Nobel Prize winners, such as Watson and Crick, who discovered the double helix of DNA. The mathematician Ramanujan was also enrolled in Trinity College at one point, and Wiles, who proved Fermat's last theorem, resided in Cambridge (see Figure 7.)

While they are aware of the pretension behind the thought that mathematics and science caused the country to flourish with prosperity, the people consider mathematics as important. In Japan on the other hand, as expressed by the words 'mathemat-

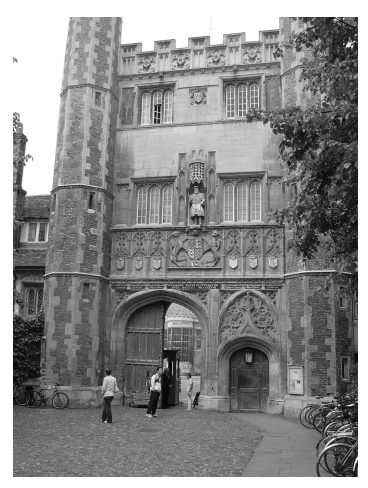

Figure 7: The main entrance of Trinity College. Newton and Ramanujan both studied here. ics for examinations', mathematics is only used as a method of sorting examinees by ability. I've presented a description somewhat biased towards the UK, but even removing this partiality, surely half of the description hits the mark.

I had the opportunity to study in Cambridge from April 2005. I graduated in mathematics in 1971, but had no postgraduate experience. Regarding research, most of my articles have been published in 'Mathematics Seminar' and deal with mathematical games. From them I had 'The Flight Mechanism of Boomerangs', 'The Puzzle of the Five Petals', and 'Constructions with Fixed Points' translated into English, and applied for overseas study. When I arrived in April, I was posted in the same relativity theory group as the astrophysicist Stephen Hawking and provided with a personal research room. Speaking in the extreme, it means to say that my article on boomerangs was acknowledged at Cambridge. I feel that the place has the fascination of an ancient university, which makes one want to try to see everything there is to see. It was a year during which I experienced the climate of the UK, where they accept Sudoku as legitimate mathematics, and I was compelled to think about the state of mathematics and mathematicians in Japan.

Eugenie Samuel Reich, Mathematician claims breakthrough in Sudoku puz- 
zle, Puzzles must have at least 17 clues to have a valid solution, Nature, 06 January 2012

http://www.nature.com/news/mathematician-claims-breakthrough-in-sudokupuzzle-1.9751

\section{References}

[1] S.E. Bammel, J. Rothstein, The Number of $9 \times 9$ Latin squares, Discrete Mathematics, 11 (1975), 93-95.

[2] B. Felgenhauer, F. Jarvis, Enumerating possible Sudoku grids (2005).

[3] G. Royle, Minimum Sudoku (2005).

[4] Sudoku article on Wikipedia, the free encyclopedia, http://en.wikipedia.org/wiki/Sudoku 